\title{
Favourite sites, favourite values and jump sizes for random walk and Brownian motion
}

\author{
ENDRE CSÁKI ${ }^{1}$, PÁL RÉVÉSZ ${ }^{2}$ and ZHAN SHI ${ }^{3}$ \\ ${ }^{1}$ Alfréd Rényi Institute of Mathematics, Hungarian Academy of Sciences, Budapest, P.O.B. 127, \\ H-1364, Hungary.E-mail: csaki@math-inst.hu \\ ${ }^{2}$ Institut für Statistik und Wahrscheinlichkeitstheorie, Technische Universität Wien, Wiedner \\ Hauptstrasse 8-10/107, A-1040 Vienna, Austria. E-mail: revesz@ci.tuwien.ac.at \\ ${ }^{3}$ Laboratoire de Probabilités, Université Paris VI, 4 Place Jussieu, F-75252 Paris Cedex 05, \\ France.E-mail: zhan@proba.jussieu.fr
}

We determine: (a) the joint almost sure asymptotic behaviour of the most visited site and the maximum local time of a one-dimensional simple random walk or Brownian motion; (b) the maximal jump size of the most visited site. In so doing, we solve two open problems of Erdős and Révész.

Keywords: Brownian motion; favourite site; local time; random walk

\section{Introduction}

Consider a one-dimensional simple symmetric random walk $\left\{S_{n}\right\}_{n \geqslant 0}$, starting from $S_{0}=0$. Let

$$
\xi(n, x)=\#\left\{0 \leqslant k \leqslant n: S_{k}=x\right\} .
$$

Thus, $\xi(n, x)$ records the number of times the random walk visits the site $x$ in the first $n$ steps. Define

$$
\mathbb{U}(n)=\left\{x \in \mathbb{Z}: \xi(n, x)=\sup _{y \in \mathbb{Z}} \xi(n, y)\right\} .
$$

In words, $\mathbb{U}(n)$ is the set of the most visited sites (or favourite sites) of the random walk at time $n$. Let

$$
U(n)=\max _{x \in \mathbb{U}(n)} x .
$$

In Erdős and Révész (1984), $U(n)$ was called the (largest) favourite site of $\left\{S_{k}\right\}_{0 \leqslant k \leqslant n}$. The choice of $U(n)$ is irrelevant in the sense that all the results for $U(n)$ stated in this paper remain true if 'max' is replaced, for example, by ' $\min$ ' in (1.2).

The study of asymptotic properties of $\mathbb{U}(n)$ and $U(n)$ was initiated by Erdös and Révész (1984) and Bass and Griffin (1985), and followed by several groups of mathematicians. 
Nevertheless, little is known so far, due mainly to the fact that the process $n \mapsto U(n)$ has some very peculiar asymptotic behaviours. For example, a surprising theorem of Bass and Griffin (1985) confirms the transience of $U(n)$, in the sense that $\lim _{n \rightarrow \infty}|U(n)|=\infty$ almost surely. However, it is an open problem to determine the rate of escape of $U(n)$. Another interesting problem is from Erdős and Révész (1984), who conjectured that almost surely for all sufficiently large $n, \mathbb{U}(n)$ contains at most two points.

We are interested in the limsup asymptotics of $U(n)$. First, we recall the law of the iterated logarithm (LIL) for $U(n)$ which was proved by Erdős and Révész (1984) and Bass and Griffin (1985), using different methods:

$$
\limsup _{n \rightarrow \infty} \frac{U(n)}{\phi(n)}=1 \text { a.s., }
$$

where

$$
\phi(n)=(2 n \log \log n)^{1 / 2}=\left(2 n \log _{2} n\right)^{1 / 2} .
$$

(However, the problem of characterizing the upper class functions of $U(n)$ via an integral test in the sense of P. Lévy remains open. For a list of ten other open problems concerning favourite sites, see Révész 1990, pp. 130-131.)

We also recall the LIL for maximum local time: with

$$
\xi^{*}(n)=\sup _{x \in \mathbb{Z}} \xi(n, x),
$$

we have

$$
\limsup _{n \rightarrow \infty} \frac{\xi^{*}(n)}{\phi(n)}=1 \text { a.s. }
$$

This was first proved by Kesten (1965), and it was extended to the form of an integral test by Csáki (1989).

In their proof of (1.3), Erdős and Révész (1984) noticed that, for any $\varepsilon>0$, almost surely there exist infinitely many $n$ such that simultaneously $U(n) \geqslant(1-\varepsilon) \phi(n)$ and $\xi^{*}(n) \geqslant c \phi(n)$, for some positive constant $c$ depending on $\varepsilon$. This led them to ask what can be said about the joint asymptotics of $U(n)$ and $\xi^{*}(n)$. When $U(n)$ is very close to its maximum possible value, how large can $\xi^{*}(n)$ be? This question also appeared later in Erdős and Révész (1987), and it was also stated as open problem 1 in Révész (1990, p. $130)$.

If $U(n)$ and $\xi^{*}(n)$ were asymptotically independent, then one would expect that the limit set of $\left\{\left(U(n) / \phi(n), \xi^{*}(n) / \phi(n)\right)\right\}$ should be $\left\{(x, y): x^{2}+y^{2} \leqslant 1\right\}$. However, things do not quite go like this. In this regard, we have the following result:

Theorem 1.1. With probability one, the random sequence

$$
\left\{\left(\frac{U(n)}{\phi(n)}, \frac{\xi^{*}(n)}{\phi(n)}\right)\right\}_{n \geqslant 3}
$$


is relatively compact, and its limit set is identical to the simplex

$$
\mathscr{C}=\{(x, y): y \geqslant 0,|x|+y \leqslant 1\} .
$$

Remark. Our Theorem 1.1 shows that favourite value and favourite site are somewhat negatively dependent, that is, if the most visited site is small then the time spent in it is large, and if it is large then the time spent in it is correspondingly smaller.

The second question we are interested in is also due to Erdős and Révész (1984; 1987), and was stated as open problem 4 in Révész (1990, p. 130): what is the maximal jump size of $U(n)$ ? The jump size of $U(n)$ is defined as $U(n+1)-U(n)$ if it does not vanish. Concerning its large values, they asked whether it is possible to determine the function $f(n)$ such that $\lim _{\sup _{n \rightarrow \infty}}(U(n+1)-U(n)) / f(n)=1$ almost surely.

In view of (1.3), one might expect $U(n+1)-U(n)$ to be a constant multiple of $\phi(n)$ along a random subsequence. What might be somewhat surprising is that it turns out to be as large as $(1-\varepsilon) \phi(n)$, for any $\varepsilon>0$.

Theorem 1.2. We have

$$
\limsup _{n \rightarrow \infty} \frac{U(n+1)-U(n)}{\phi(n)}=1 \text { a.s. }
$$

In the study of favourite sites, it is more frequent to encounter questions than answers, and this may help explain why there are relatively few known results compared with the huge literature on random walks. We mention the work of Tóth and Werner (1997) who were interested in favourite edges instead of favourite sites, and of Csáki and Shi (1998) who studied how close a favourite site can be to the frontier of the range of a random walk.

It will be clear from the proofs in Sections 4 and 5 that both Theorems 1.1 and 1.2 have their counterparts for Brownian motion, which we state as follows. Similarly to the case of random walks, we can define the set of favourite sites of Brownian motion as

$$
\mathbb{V}(t)=\left\{x \in \mathbb{R}: L(t, x)=\sup _{y \in \mathbb{R}} L(t, y)\right\},
$$

where $L$ denotes the local time of a Brownian motion. It is known (Eisenbaum 1989; 1997; Leuridan 1997) that, with probability one, $\mathbb{V}(t)$ contains at most two points for all $t>0$. Without loss of generality, let us choose

$$
V(t)=\max _{x \in \mathbb{V}(t)} x .
$$

According to Eisenbaum (1989), $\{V(t) ; t>0\}$ is a process with limits on the left almost surely.

Theorem 1.3. Let $L^{*}(t)=\sup _{x \in \mathbb{R}} L(t, x)$. The limit set of $\left\{\left(V(t) / \phi(t), L^{*}(t) / \phi(t)\right)\right\}_{t \geqslant 3}$ is almost surely $\{(x, y): y \geqslant 0,|x|+y \leqslant 1\}$. 
Theorem 1.4. We have

$$
\limsup _{t \rightarrow \infty} \frac{V(t)-V(t-)}{\phi(t)}=1 \text { a.s. }
$$

Let us say a few words about the organization of this paper. Looking at Theorem 1.1, that $\left\{\left(U(n) / \phi(n), \xi^{*}(n) / \phi(n)\right)\right\}_{n \geqslant 3}$ is almost surely relatively compact follows from (1.3) and (1.6), so we only have to show that its limit set equals $\mathscr{C}$. This is done in two steps. Namely, we prove that, almost surely,

(a) any $(x, y) \in \mathscr{C}$ is a limit point of $\left\{\left(U(n) / \phi(n), \xi^{*}(n) / \phi(n)\right)\right\}_{n \geqslant 3}$;

(b) all the limit points of $\left\{\left(U(n) / \phi(n), \xi^{*}(n) / \phi(n)\right)\right\}_{n \geqslant 3}$ are contained in $\mathscr{A}$.

Parts (a) and (b) are proved in Sections 4 and 5 respectively; furthermore, Section 4 contains the proof of Theorem 1.2. Before that, in Section 2 we present some probability estimates for multidimensional Brownian motion, which may be of independent interest. These estimates are applied to Brownian local times in Section 3, and enable us to obtain the key ingredient in the proofs of the theorems.

We conclude this Introduction with a brief remark on notation. Throughout this paper, $a(x) \sim b(x)\left(x \rightarrow x_{0}\right)$ denotes $\lim _{x \rightarrow x_{0}} a(x) / b(x)=1$ for positive $a(x)$ and $b(x)$.

\section{Probability estimates for Brownian motion}

This section contains some probability estimates for $d$-dimensional Brownian motion. Although only the special case $d=2$ is needed in the proof of the theorems, we present the estimates for any dimension $d \geqslant 1$.

In the rest of the section, $\{B(t) ; t \geqslant 0\}$ denotes an $\mathbb{R}^{d}$-valued Brownian motion $(d \geqslant 1)$ and

$$
R(t)=\|B(t)\|, \quad t \geqslant 0,
$$

is the Euclidean modulus of $B$. Note that $R$ is a $d$-dimensional Bessel process. For $x \geqslant 0$, let $\mathbb{P}^{x}$ denote the probability measure under which $R$ starts from $x$. We assume that under $\mathbb{P}$, $R(0)=0$. Thus $\mathbb{P}^{0}=\mathbb{P}$. We also write $\mathbb{E}^{x}$ for the expectation with respect to $\mathbb{P}^{x}$.

The square integral of $R$ plays an important role in our approach. We write, for brevity,

$$
X(t)=\int_{0}^{t} R^{2}(s) \mathrm{d} s, \quad t \geqslant 0 .
$$

We start by recalling two useful results. The first is an intuitively clear comparison criterion for $R$. It can easily be proved by applying the diffusion comparison theorem stated in Revuz and Yor (1999, Theorem IX.3.7) to squared Bessel processes. The second is a special case of de Bruijn's exponential-type Tauberian theorem (see Bingham et al. 1987, p. 254). 
Fact 2.1. Let $0 \leqslant x<y$. The Bessel process $\{R(t) ; t \geqslant 0\}$ is stochastically smaller under $\mathbb{P}^{x}$ than under $\mathbb{P}^{y}$.

Fact 2.2. Let $\Lambda$ be a random variable with $\mathbb{P}(\Lambda \geqslant 0)=1$, and let $a>0$ be a constant. The following conditions are equivalent:

$$
\begin{aligned}
\lim _{r \rightarrow \infty} \frac{1}{\sqrt{r}} \log \mathbb{E}\left(\mathrm{e}^{-r \Lambda}\right) & =-a, \\
\lim _{\varepsilon \rightarrow 0} \varepsilon \log \mathbb{P}(\Lambda<\varepsilon) & =-\frac{a^{2}}{4} .
\end{aligned}
$$

We now prove a few preliminary estimates.

Lemma 2.3. Fix $\theta \in(0,1), t>0$ and $r>r_{1}>0$. Then

$$
\lim _{\varepsilon \rightarrow 0} \varepsilon \log \mathbb{P}\left(\theta \varepsilon \leqslant X(t) \leqslant \varepsilon, r_{1} \leqslant R(t) \leqslant r\right)=-\frac{\left(r_{1}^{2}+t d\right)^{2}}{8} .
$$

Proof. The conditional Laplace transform of $X(t)$ given $(R(0), R(t))$ is known (cf. Pitman and Yor 1982):

$\mathbb{E}^{x}\left(\mathrm{e}^{-\left(\lambda^{2} / 2\right) X(t)} \mid R(t)=y\right)=\frac{\lambda t}{\sinh (\lambda t)} \frac{I_{(d-2) / 2}(\lambda x y / \sinh (\lambda t))}{I_{(d-2) / 2}(x y / t)} \exp \left(-\frac{\left(x^{2}+y^{2}\right)(\lambda t \operatorname{coth}(\lambda t)-1)}{2 t}\right)$,

where $I_{(d-2) / 2}(\cdot)$ denotes the modified Bessel function of index $(d-2) / 2$. For convenience, we recall here the asymptotics of the modified Bessel function (cf. Abramowitz and Stegun 1965, pp. 375 and 377, respectively):

$$
\begin{aligned}
& I_{p}(z) \sim(z / 2)^{p} / \Gamma(p+1), \quad z \rightarrow 0, \\
& I_{p}(z) \sim \mathrm{e}^{z} / \sqrt{2 \pi z}, \quad z \rightarrow \infty .
\end{aligned}
$$

In view of (2.3), we can let $x$ go to 0 in (2.2) to see that

$$
\mathbb{E}\left(\mathrm{e}^{\left(\lambda^{2} / 2\right) X(t)} \mid R(t)=y\right)=\left(\frac{\lambda t}{\sinh (\lambda t)}\right)^{d / 2} \exp \left(-\frac{y^{2}(\lambda t \operatorname{coth}(\lambda t)-1)}{2 t}\right) .
$$

Thus,

$$
\begin{aligned}
& \mathbb{E}\left(\mathrm{e}^{-\left(\lambda^{2} / 2\right) X(t)} \mid r_{1} \leqslant R(t) \leqslant r\right) \\
& \quad=\int_{r_{1}}^{r} \frac{\mathbb{P}(R(t) \in \mathrm{d} y)}{\mathbb{P}\left(r_{1} \leqslant R(t) \leqslant r\right)}\left(\frac{\lambda t}{\sinh (\lambda t)}\right)^{d / 2} \exp \left(-\frac{y^{2}(\lambda t \operatorname{coth}(\lambda t)-1)}{2 t}\right) .
\end{aligned}
$$

When $\lambda \rightarrow \infty$, the expression on the right-hand side is $\exp \left(-(1+o(1))\left(r_{1}^{2}+t d\right) \lambda / 2\right)$. Applying de Bruijn's Tauberian theorem (Fact 2.2) gives that, as $\varepsilon \rightarrow 0$, 


$$
\mathbb{P}\left(X(t)<\varepsilon \mid r_{1} \leqslant R(t) \leqslant r\right)=\exp \left(-(1+o(1)) \frac{\left(r_{1}^{2}+t d\right)^{2}}{8 \varepsilon}\right)
$$

Therefore,

$$
\mathbb{P}\left(X(t)<\varepsilon, r_{1} \leqslant R(t) \leqslant r\right)=\exp \left(-(1+o(1)) \frac{\left(r_{1}^{2}+t d\right)^{2}}{8 \varepsilon}\right) .
$$

Similarly, using (2.5) and Fact 2.2, we have that, for any $y>0$, when $\varepsilon \rightarrow 0$,

$$
\mathbb{P}(X(t)<\varepsilon \mid R(t)=y)=\exp \left(-(1+o(1)) \frac{\left(y^{2}+t d\right)^{2}}{8 \varepsilon}\right) .
$$

This estimate will be of use later.

The probability expression on the left-hand side of (2.1) can be written as

$$
\mathbb{P}\left(X(t)<\varepsilon, r_{1} \leqslant R(t) \leqslant r\right)-\mathbb{P}\left(X(t)<\theta \varepsilon, r_{1} \leqslant R(t) \leqslant r\right) .
$$

In view of (2.6), this yields Lemma 2.3 .

Lemma 2.4. For any $x>0$,

$$
\mathbb{P}^{x}(X(t)<\varepsilon)=\exp \left(-(1+o(1)) \frac{\left(x^{2}+t d\right)^{2}}{8 \varepsilon}\right), \quad \varepsilon \rightarrow 0 .
$$

Proof. Recall the probability transition density of $R$ (see Revuz and Yor 1999, Chapter XI): for $y>0$,

$$
\mathbb{P}^{x}(R(t) \in \mathrm{d} y)=\frac{y}{t}\left(\frac{y}{x}\right)^{(d-2) / 2} I_{(d-2) / 2}\left(\frac{x y}{t}\right) \exp \left(-\frac{x^{2}+y^{2}}{2 t}\right) \mathrm{d} y .
$$

Writing

$$
\mathbb{E}^{x}\left(\mathrm{e}^{-\left(\lambda^{2} / 2\right) X(t)}\right)=\int_{0}^{\infty} \mathbb{P}^{x}(R(t) \in \mathrm{d} y) \mathbb{E}^{x}\left(\mathrm{e}^{-\left(\lambda^{2} / 2\right) X(t)} \mid R(t)=y\right),
$$

and using (2.2)-(2.4) and (2.9), we easily arrive at

$$
\log \mathbb{E}^{x}\left(\mathrm{e}^{-\left(\lambda^{2} / 2\right) X(t)}\right)=-(1+o(1)) \frac{\left(x^{2}+t d\right) \lambda}{2}, \quad \lambda \rightarrow \infty .
$$

Lemma 2.4 now follows from an application of Fact 2.2.

Lemma 2.5. Fix $\theta \in(0,1), t>0, v>\frac{1}{2}$ and $r>r_{1}>0$. Assuming

$$
\left(r^{2}+t d\right)^{2} \theta<\left(r_{1}^{2}+t d\right)^{2},
$$

we have

$$
\lim _{\varepsilon \rightarrow 0} \inf _{x \in\left[r_{1}, r\right]} \varepsilon \log \mathbb{P}^{x}\left(\theta \varepsilon \leqslant X(t) \leqslant \varepsilon, R(t)<\varepsilon^{v}\right)=-\frac{\left(r^{2}+t d\right)^{2}}{8}
$$


Proof. Recall that $R(t)=\|B(t)\|$. Under $\mathbb{P}^{x}, B$ can be realized as an $\mathbb{R}^{d}$-valued Brownian motion with $\|B(0)\|=x$. Define $B_{0}(s)=B(s)-(s / t) B(t)$ (for $s \in[0, t]$ ), which is a Brownian bridge on $[0, t]$, independent of $B(t)$. Let $R_{0}(s)=\left\|B_{0}(s)\right\|$. Note that $\left\{R_{0}(s) ; s \in[0, t]\right\}$ is a so-called Bessel bridge starting from $x$ (under $\mathbb{P}^{x}$ ), 'conditioned to hit 0 at time $t^{\prime}$, independent of $R(t)$.

By the triangular inequality, $\left|R(s)-R_{0}(s)\right| \leqslant R(t)$ for all $s \in[0, t]$. By Minkowski's inequality, $\left|\sqrt{X(t)}-\left(\int_{0}^{t} R_{0}^{2}(s) \mathrm{d} s\right)^{1 / 2}\right| \leqslant \sqrt{t} R(t)$. Therefore, for any $y>0$ and $q>0$,

$$
\begin{aligned}
\mathbb{P}^{x}(X(t) \leqslant q \mid R(t)=y) & \geqslant \mathbb{P}^{x}\left(\left(\int_{0}^{t} R_{0}^{2}(s) \mathrm{d} s\right)^{1 / 2} \leqslant \sqrt{q}-\sqrt{t} y\right) \\
& =\mathbb{P}(\sqrt{X(t)} \leqslant \sqrt{q}-\sqrt{t} y \mid R(t)=x),
\end{aligned}
$$

the last equality following from the time inversion: $\left\{R_{0}(t-s) ; s \in[0, t]\right\}$ is again a Bessel bridge, starting from 0 , conditioned to hit $x$ at time $t$. Hence, by Fact 2.1, when $\varepsilon$ is so small that $\sqrt{t} \varepsilon^{v}<\sqrt{\varepsilon}$

$$
\begin{aligned}
\inf _{x \in\left[r_{1}, r\right]} \mathbb{P}^{x}\left(X(t) \leqslant \varepsilon, R(t)<\varepsilon^{\nu}\right) & =\mathbb{P}^{r}\left(X(t) \leqslant \varepsilon, R(t)<\varepsilon^{\nu}\right) \\
& \geqslant \mathbb{P}^{r}\left(R(t) \leqslant \varepsilon^{\nu}\right) \mathbb{P}\left(X(t) \leqslant\left(\sqrt{\varepsilon}-\sqrt{t} \varepsilon^{\nu}\right)^{2} \mid R(t)=r\right) .
\end{aligned}
$$

By (2.7), when $\varepsilon \rightarrow 0$, the second probability term on the right-hand side of (2.11) is $\exp \left(-(1+o(1))\left(r^{2}+t d\right)^{2} / 8 \varepsilon\right)$, whereas $\mathbb{P}^{r}\left(R(t)<\varepsilon^{\nu}\right)$ is of polynomial order of $\varepsilon^{v}$ (this is an immediate consequence of (2.9) and (2.3), or can be better understood using the fact that $R^{2}(t)$ under $\mathbb{P}^{r}$ has a non-centred chi-square law). Consequently,

$$
\liminf _{\varepsilon \rightarrow 0} \inf _{x \in\left[r_{1}, r\right]} \varepsilon \log \mathbb{P}^{x}\left(X(t) \leqslant \varepsilon, R(t)<\varepsilon^{\nu}\right) \geqslant-\frac{\left(r^{2}+t d\right)^{2}}{8}
$$

On the other hand, by Fact 2.1,

$$
\begin{aligned}
\sup _{x \in\left[r_{1}, r\right]} \mathbb{P}^{x}\left(X(t)<\theta \varepsilon, R(t)<\varepsilon^{v}\right) & \leqslant \mathbb{P}^{r_{1}}(X(t)<\theta \varepsilon) \\
& =\exp \left(-(1+o(1)) \frac{\left(r_{1}^{2}+t d\right)^{2}}{8 \theta \varepsilon}\right),
\end{aligned}
$$

the last identity following from (2.8). Combining (2.12) with (2.13), and in view of (2.10), we obtain the lower bound in the lemma.

The upper bound is easy. Indeed, just as for (2.13), we have

$$
\begin{aligned}
\inf _{x \in\left[r_{1}, r\right]} \mathbb{P}^{x}\left(\theta \varepsilon \leqslant X(t) \leqslant \varepsilon, R(t)<\varepsilon^{v}\right) & \leqslant \mathbb{P}^{r}(X(t) \leqslant \varepsilon) \\
& =\exp \left(-(1+o(1)) \frac{\left(r^{2}+t d\right)^{2}}{8 \varepsilon}\right),
\end{aligned}
$$

as desired. 
Lemma 2.6. For $r>0$ and $t>0$,

$$
\limsup _{\varepsilon \rightarrow 0} \sup _{x \in[0, r]} \varepsilon \log \mathbb{P}^{x}\left(X(t)<\varepsilon, \sup _{0 \leqslant s \leqslant t} R(s) \geqslant r\right) \leqslant-\frac{\left(r^{2}+t d\right)^{2}}{8} .
$$

Proof. Let

$$
H(r)=\inf \{t>0: R(t)>r\} .
$$

For each $x \in[0, r]$, the probability term on the left-hand side of (2.14) is

$$
\leqslant \mathbb{P}^{x}(H(r) \leqslant t, X(t)-X(H(r))<\varepsilon) \leqslant \mathbb{P}^{r}(X(t)<\varepsilon),
$$

the second inequality following from the strong Markov property. Now (2.14) follows from Lemma 2.4.

Lemma 2.7. Let $0<x_{1}<x_{2}<r_{1}^{*}<r_{2}^{*}$ and $r_{1}<r_{2}<r_{1}^{*}$ be such that

$$
r_{2}^{*}-r_{1}^{*}>r_{1}^{*}-r_{1}
$$

Then

$$
\liminf _{t \rightarrow 0} \inf _{x \in\left[x_{1}, x_{2}\right]} t \log \mathbb{P}^{x}\left(\sup _{0 \leqslant s \leqslant t} R(s) \in\left[r_{1}^{*}, r_{2}^{*}\right], R(t) \in\left[r_{1}, r_{2}\right]\right) \geqslant-\left(r_{1}^{*}-x_{1}\right)^{2}-\left(r_{1}^{*}-r_{1}\right)^{2} .
$$

Proof. We first recall the tail estimate of $R$, which follows from a well-known tail estimate for general Gaussian processes (see, for example, Marcus and Shepp 1972): for $r>0$,

$$
\mathbb{P}\left(\sup _{0 \leqslant s \leqslant t} R(s)>r\right)=\exp \left(\left(-(1+o(1)) \frac{r^{2}}{2 t}\right), \quad t \rightarrow 0 .\right.
$$

Now look at the probability

$$
\mathbb{P}^{r_{1}^{*}}\left(R(t) \in\left[r_{1}, r_{2}\right]\right)=\int_{r_{1}}^{r_{2}} \mathbb{P}_{1}^{*}(R(t) \in \mathrm{d} y)
$$

From (2.9) and (2.4), it is easily seen that for $y$ and $r$ bounded (from above and below), and for $t \in(0,1]$,

$$
\frac{\mathbb{P}^{r}(R(t) \in \mathrm{d} y)}{\mathrm{d} y} \geqslant \frac{c_{1}}{\sqrt{t}} \exp \left(-\frac{(r-y)^{2}}{2 t}\right),
$$

where $c_{1}>0$ is a constant depending only on $d$ and on the bounds for $(y, r)$. Thus, for $t \in(0,1]$,

$$
\mathbb{P}^{r_{1}^{*}}\left(R(t) \in\left[r_{1}, r_{2}\right]\right) \geqslant \frac{c_{1}\left(r_{2}-r_{1}\right)}{\sqrt{t}} \exp \left(-\frac{\left(r_{1}^{*}-r_{1}\right)^{2}}{2 t}\right) .
$$

On the other hand, under $\mathbb{P}^{r_{1}^{*}}, R$ is the modulus of an $\mathbb{R}^{d}$-valued Brownian motion $B$ with $\|B(0)\|=r_{1}^{*}$. By the triangular inequality, 
Favourite sites, favourite values and jump sizes

$$
\mathbb{P}^{r_{1}^{*}}\left(\sup _{0 \leqslant s \leqslant t} R(s)>r_{2}^{*}\right) \leqslant \mathbb{P}\left(\sup _{0 \leqslant s \leqslant t} R(s)>r_{2}^{*}-r_{1}^{*}\right)
$$

which, according to (2.17), is $\exp \left(-(1+o(1))\left(r_{2}^{*}-r_{1}^{*}\right)^{2} / 2 t\right)$, when $t$ goes to 0 . This, together with (2.19) and (2.16), implies that

$$
\mathbb{P}^{r_{1}^{*}}\left(\sup _{0 \leqslant s \leqslant t} R(s) \leqslant r_{2}^{*}, R(t) \in\left[r_{1}, r_{2}\right]\right) \geqslant \exp \left(-(1+o(1)) \frac{\left(r_{1}^{*}-r_{1}\right)^{2}}{2 t}\right)
$$

when $t$ goes to 0 .

Recall $H\left(r_{1}^{*}\right)$ from (2.15). For $x \in\left[x_{1}, x_{2}\right]$, we have, by the strong Markov property,

$$
\begin{aligned}
\mathbb{P}^{x}\left(\sup _{0 \leqslant s \leqslant t} R(s) \in\left[r_{1}^{*}, r_{2}^{*}\right], R(t) \in\left[r_{1}, r_{2}\right]\right) \\
=\mathbb{P}^{x}\left(H\left(r_{1}^{*}\right) \leqslant t, \sup _{0 \leqslant s \leqslant t} R(s) \leqslant r_{2}^{*}, R(t) \in\left[r_{1}, r_{2}\right]\right) \\
=\int_{0}^{t} \mathbb{P}^{x}\left(H\left(r_{1}^{*}\right) \in \mathrm{d} u\right) \mathbb{P}^{r_{1}^{*}}\left(\sup _{0 \leqslant s \leqslant t-u} R(s) \leqslant r_{2}^{*}, R(t-u) \in\left[r_{1}, r_{2}\right]\right) \\
\geqslant \int_{0}^{t / 2} \mathbb{P}^{x}\left(H\left(r_{1}^{*}\right) \in \mathrm{d} u\right) \mathbb{P}^{r_{1}^{*}}\left(\sup _{0 \leqslant s \leqslant t-u} R(s) \leqslant r_{2}^{*}, R(t-u) \in\left[r_{1}, r_{2}\right]\right) \\
\geqslant \int_{0}^{t / 2} \mathbb{P}^{x}\left(H\left(r_{1}^{*}\right) \in \mathrm{d} u\right) \exp \left(-(1+o(1)) \frac{\left(r_{1}^{*}-r_{1}\right)^{2}}{t}\right),
\end{aligned}
$$

the last equality following from (2.20), where $o(1)$ is uniform in $x \in\left[x_{1}, x_{2}\right]$. Lemma 2.7 will be proved if we can verify

$$
\inf _{x \in\left[x_{1}, x_{2}\right]} \mathbb{P}^{x}\left(H\left(r_{1}^{*}\right) \leqslant t / 2\right) \geqslant \exp \left(-(1+o(1)) \frac{\left(r_{1}^{*}-x_{1}\right)^{2}}{t}\right), \quad t \rightarrow 0 .
$$

To this end, observe that, for $x \in\left[x_{1}, x_{2}\right]$,

$$
\mathbb{P}^{x}\left(H\left(r_{1}^{*}\right) \leqslant t / 2\right) \geqslant \mathbb{P}^{x}\left(R(t / 2)>r_{1}^{*}\right)=\int_{r_{1}^{*}}^{\infty} \mathbb{P}^{x}(R(t / 2) \in \mathrm{d} y)
$$

By (2.18), there exists a constant $c_{1}$ depending only on $\left(r_{1}^{*}, r_{2}^{*}, x_{1}, x_{2}, d\right)$, such that, for all $x \in\left[x_{1}, x_{2}\right]$, 


$$
\begin{aligned}
\mathbb{P}^{x}\left(H\left(r_{1}^{*}\right) \leqslant t / 2\right) & \geqslant \int_{r_{1}^{*}}^{r_{2}^{*}} \mathbb{P}^{x}(R(t / 2) \in \mathrm{d} y) \\
& \geqslant \frac{2 c_{1}}{t} \int_{r_{1}^{*}}^{r_{2}^{*}} \exp \left(-\frac{(y-x)^{2}}{t}\right) \mathrm{d} y \\
& \geqslant \frac{2 c_{1}}{t} \int_{r_{1}^{*}}^{r_{2}^{*}} \exp \left(-\frac{\left(y-x_{1}\right)^{2}}{t}\right) \mathrm{d} y \\
& =\exp \left((1+o(1)) \frac{\left(r_{1}^{*}-x_{1}\right)^{2}}{t}\right),
\end{aligned}
$$

which yields (2.21) and thus completes the proof of Lemma 2.7 .

Lemma 2.8. Let $a \in(0,1), b_{2}>b_{1}>\delta>0, \rho>1, v>\frac{1}{2}$ and $0<c<(\rho-1) / b_{2}$. Assume

$$
\sqrt{b_{2}}-\sqrt{b_{1}}>\sqrt{b_{1}}-\sqrt{b_{1}-\delta} \text {. }
$$

Let

$$
\begin{gathered}
E_{1}(\varepsilon)=\left\{\varepsilon \leqslant X(1+c \varepsilon) \leqslant \rho \varepsilon, R(1+c \varepsilon)<\varepsilon^{v}, b_{1} \leqslant \sup _{s \in[a, a+c \varepsilon]} R^{2}(s) \leqslant b_{2},\right. \\
\left.\sup _{s \in[a, a+c \varepsilon]} R^{2}(s) \geqslant \delta+\sup _{s \in[0,1+c \varepsilon] \backslash[a, a+c \varepsilon]} R^{2}(s)\right\} .
\end{gathered}
$$

Then

$$
\liminf _{\varepsilon \rightarrow 0} \varepsilon \log \mathbb{P}\left(E_{1}(\varepsilon)\right) \geqslant-\frac{\left(2\left(b_{1}-\delta\right)+d\right)^{2}}{8}-\frac{5\left(\sqrt{b_{1}}-\sqrt{b_{1}-\delta}\right)^{2}}{c} .
$$

Proof. Fix $r \in\left(0, \sqrt{b_{1}-\delta}\right)$ such that $\sqrt{b_{2}}-\sqrt{b_{1}}>\sqrt{b_{1}}-r$. Define

$$
\beta=\frac{r^{2}+(1-a) d}{2 r^{2}+d} \text {. }
$$

Since $\beta \in(0,1)$ and $c<(\rho-1) / b_{2}$, it is possible to choose $0<\beta_{*}<\beta<\beta^{*}<1$ such that

$$
1-\beta_{*}+b_{2} c+\beta^{*} \leqslant \rho .
$$

Let $r^{*} \in\left(r, \sqrt{b_{1}-\delta}\right)$. We also choose $r_{*} \in(0, r)$ so that

$$
\begin{gathered}
\sqrt{b_{1}}-r_{*} \leqslant 2\left(\sqrt{b_{1}}-r\right), \\
\sqrt{b_{1}}-r_{*}<\sqrt{b_{2}}-\sqrt{b_{1}}, \\
\left(r^{2}+(1-a) d\right)^{2} \frac{\beta}{\beta^{*}}<\left(r_{*}^{2}+(1-a) d\right)^{2} .
\end{gathered}
$$


Favourite sites, favourite values and jump sizes

Consider the measurable events

$$
\begin{aligned}
& E_{2}(\varepsilon)=\left\{(1-\beta) \varepsilon \leqslant X(a) \leqslant\left(1-\beta_{*}\right) \varepsilon, \sup _{0 \leqslant s \leqslant a} R(s)<\sqrt{b_{1}-\delta}, r \leqslant R(a) \leqslant r^{*}\right\}, \\
& E_{3}(\varepsilon)=\left\{\sqrt{b_{1}} \leqslant \sup _{a \leqslant s \leqslant a+c \varepsilon} R(s) \leqslant \sqrt{b_{2}}, r_{*} \leqslant R(a+c \varepsilon) \leqslant r\right\}, \\
& E_{4}(\varepsilon)=\left\{\beta \varepsilon \leqslant X(1+c \varepsilon)-X(a+c \varepsilon) \leqslant \beta^{*} \varepsilon, \sup _{a+c \varepsilon \leqslant s \leqslant 1+c \varepsilon} R(s) \leqslant \sqrt{b_{1}-\delta}, R(1+c \varepsilon)<\varepsilon^{v}\right\} .
\end{aligned}
$$

Observe that on $E_{3}(\varepsilon)$ we have $X(a+c \varepsilon)-X(a) \in\left[0, b_{2} c \varepsilon\right]$. In view of (2.23), on $E_{2}(\varepsilon) \cap E_{3}(\varepsilon) \cap E_{4}(\varepsilon)$, we have $X(1+c \varepsilon) \in[\varepsilon, \rho \varepsilon]$. Thus

$$
E_{1}(\varepsilon) \supset E_{2}(\varepsilon) \cap E_{3}(\varepsilon) \cap E_{4}(\varepsilon),
$$

By the Markov property,

$$
\begin{aligned}
\mathbb{P}\left(E_{1}(\varepsilon)\right) & \geqslant \mathbb{P}\left(E_{2}(\varepsilon) \cap E_{3}(\varepsilon) \cap E_{4}(\varepsilon)\right) \\
& \geqslant \mathbb{P}\left(E_{2}(\varepsilon)\right) \inf _{x \in\left[r, r^{*}\right]} p_{1}(x, \varepsilon) \inf _{y \in\left[r_{*}, r\right]} p_{2}(y, \varepsilon),
\end{aligned}
$$

where, for $x \in\left[r, r^{*}\right]$ and $y \in[r *, r]$,

$$
\begin{aligned}
& p_{1}(x, \varepsilon)=\mathbb{P}^{x}\left(\sqrt{b_{1}} \leqslant \sup _{0 \leqslant s \leqslant c \varepsilon} R(s) \leqslant \sqrt{b_{2}}, r_{*} \leqslant R(c \varepsilon) \leqslant r\right), \\
& p_{2}(y, \varepsilon)=\mathbb{P}^{y}\left(\beta \varepsilon \leqslant X(1-a) \leqslant \beta^{*} \varepsilon, \sup _{0 \leqslant s \leqslant 1-a} R(s) \leqslant \sqrt{b_{1}-\delta}, R(1-a)<\varepsilon^{v}\right) .
\end{aligned}
$$

We now estimate $\mathbb{P}\left(E_{2}(\varepsilon)\right), p_{1}(x, \varepsilon)$ and $p_{2}(y, \varepsilon)$, respectively. First, since

$$
\begin{aligned}
\mathbb{P}\left(E_{2}(\varepsilon)\right) \geqslant & \mathbb{P}\left((1-\beta) \varepsilon \leqslant X(a) \leqslant\left(1-\beta_{*}\right) \varepsilon, r \leqslant R(a) \leqslant r^{*}\right) \\
& -\mathbb{P}\left(X(a) \leqslant\left(1-\beta_{*}\right) \varepsilon, \sup _{0 \leqslant s \leqslant a} R(s) \geqslant \sqrt{b_{1}-\delta}\right),
\end{aligned}
$$

it follows from Lemmas 2.3 and 2.6 that (noting that $b_{1}-\delta>r^{2}$ )

$$
\mathbb{P}\left(E_{2}(\varepsilon)\right) \geqslant \exp \left(-(1+o(1)) \frac{\left(r^{2}+a d\right)^{2}}{8\left(1-\beta_{*}\right) \varepsilon}\right), \quad \varepsilon \rightarrow 0 .
$$

It is easy to treat $p_{1}(x, \varepsilon)$. Indeed, in view of (2.25), we can apply Lemma 2.7 to see that, when $\varepsilon \rightarrow 0$,

$$
\inf _{x \in\left[r, r^{*}\right]} p_{1}(x, \varepsilon) \geqslant \exp \left(-(1+o(1)) \frac{\left(\sqrt{b_{1}}-r\right)^{2}+\left(\sqrt{b_{1}}-r_{*}\right)^{2}}{c \varepsilon}\right) .
$$

Finally, we observe that, for all $y \in\left[r_{*}, r\right]$, 


$$
\begin{aligned}
p_{2}(y, \varepsilon) \geqslant & \inf _{y \in\left[r_{*}, r\right]} \mathbb{P}^{y}\left(\beta \varepsilon \leqslant X(1-a) \leqslant \beta^{*} \varepsilon, R(1-a)<\varepsilon^{v}\right) \\
& -\sup _{y \in\left[0, \sqrt{b_{1}-\delta}\right]} \mathbb{P}^{y}\left(X(1-a) \leqslant \beta^{*} \varepsilon, \sup _{0 \leqslant s \leqslant 1-a} R(s)>\sqrt{b_{1}-\delta}\right) .
\end{aligned}
$$

Recall (2.26). Applying Lemmas 2.5 and 2.6 yields (recalling $b_{1}-\delta>r^{2}$ ) that, when $\varepsilon \rightarrow 0$,

$$
\inf _{y \in\left[r_{*}, r\right]} p_{2}(y, \varepsilon) \geqslant \exp \left(-(1+o(1)) \frac{\left(r^{2}+(1-a) d\right)^{2}}{8 \beta^{*} \varepsilon}\right) .
$$

Assembling (2.27)-(2.30) gives

$$
\begin{aligned}
\liminf _{\varepsilon \rightarrow 0} \varepsilon \log \mathbb{P}\left(E_{1}(\varepsilon)\right) & \geqslant-\frac{\left(r^{2}+a d\right)^{2}}{8\left(1-\beta_{*}\right)}-\frac{\left(\sqrt{b_{1}}-r\right)^{2}+\left(\sqrt{b_{1}}-r_{*}\right)^{2}}{c}-\frac{\left(r^{2}+(1-a) d\right)^{2}}{8 \beta^{*}} \\
& \geqslant-\frac{\left(r^{2}+a d\right)^{2}}{8(1-\beta)}-\frac{5\left(\sqrt{b_{1}}-r\right)^{2}}{c}-\frac{\left(r^{2}+(1-a) d\right)^{2}}{8 \beta}
\end{aligned}
$$

where in the last inequality we have used $\beta^{*}>\beta>\beta_{*}$ and the relation (2.24). In view of (2.22), we obtain:

$$
\liminf _{\varepsilon \rightarrow 0} \varepsilon \log \mathbb{P}\left(E_{1}(\varepsilon)\right) \geqslant-\frac{\left(2 r^{2}+d\right)^{2}}{8}-\frac{5\left(\sqrt{b_{1}}-r\right)^{2}}{c} .
$$

Since $r$ can be as close to $\sqrt{b_{1}-\delta}$ as possible, this completes the proof of Lemma 2.8 .

Here is the main estimate which will be used in the proof of Theorem 1.1.

Lemma 2.9. Let $a \in(0,1), b_{2}>b_{1}>0, \rho>1, v>\frac{1}{2}$ and $0<c<(\rho-1) / b_{2}$. Let $\delta \in\left(0, b_{1}\right)$ be such that

$$
\sqrt{b_{2}}-\sqrt{b_{1}}>\sqrt{b_{1}}-\sqrt{b_{1}-\delta}
$$

Then

$$
\liminf _{\varepsilon \rightarrow 0} \varepsilon \log \mathbb{P}\left(E_{5}(\varepsilon)\right) \geqslant-\frac{\left(2\left(b_{1}-\delta\right)+d\right)^{2}}{8}-\frac{5\left(\sqrt{b_{1}}-\sqrt{b_{1}-\delta}\right)^{2}}{c}
$$

where

$$
\begin{gathered}
E_{5}(\varepsilon)=\left\{\varepsilon \leqslant X(1) \leqslant \rho \varepsilon, R(1)<\varepsilon^{v}, b_{1} \leqslant \sup _{s \in[0,1]} R^{2}(s) \leqslant b_{2},\right. \\
\left.\sup _{s \in[a, a+c \varepsilon]} R^{2}(s) \geqslant \delta+\sup _{s \in[0,1] \backslash[a, a+c \varepsilon]} R^{2}(s)\right\} .
\end{gathered}
$$


Proof. Let $E_{1}(\varepsilon)$ be the measurable event defined in Lemma 2.8. Note that on $E_{1}(\varepsilon)$ we have $\sup _{s \in[a, a+c \varepsilon]} R^{2}(s)=\sup _{s \in[0,1+c \varepsilon]} R^{2}(s)$. By the scaling property,

$$
\begin{aligned}
\mathbb{P}\left(E_{1}(\varepsilon)\right)=\mathbb{P}\left(\frac{\varepsilon}{(1+c \varepsilon)^{2}} \leqslant X(1) \leqslant \frac{\rho \varepsilon}{(1+c \varepsilon)^{2}}, R(1)<\frac{\varepsilon^{v}}{(1+c \varepsilon)^{1 / 2}},\right. \\
\left.\frac{b_{1}}{1+c \varepsilon} \leqslant \sup _{0 \leqslant s \leqslant 1} R^{2}(s) \leqslant \frac{b_{2}}{1+c \varepsilon}, \sup _{s \in I(\varepsilon)} R^{2}(s) \geqslant \frac{\delta}{1+c \varepsilon}+\sup _{s \in[0,1] \backslash I(\varepsilon)} R^{2}(s)\right),
\end{aligned}
$$

where $I(\varepsilon)=[a /(1+c \varepsilon),(a+c \varepsilon) /(1+c \varepsilon)]$. Applying Lemma 2.8 gives $(2.31)$.

The proof of Theorem 1.2 requires a few more probability estimates.

Lemma 2.10. For $r>0, v>\frac{1}{2}$ and $t>0$,

$$
\liminf _{\varepsilon \rightarrow 0} \inf _{x \in[0, r]} \varepsilon \log \mathbb{P}^{x}\left(\sup _{0 \leqslant s \leqslant t} R(s)>r, X(t)<\varepsilon, R(t)<\varepsilon^{v}\right) \geqslant-\frac{\left(3 r^{2}+t d\right)^{2}}{8} .
$$

Proof. Let

$$
p_{3}(x, \varepsilon)=\mathbb{P}^{x}\left(\sup _{0 \leqslant s \leqslant t} R(s)>r, X(t)<\varepsilon, R(t)<\varepsilon^{v}\right) .
$$

Recall the hitting time $H(\cdot)$ from (2.15). For any $x \in[0, r]$ and any $u \in[0, t]$,

$$
\begin{aligned}
p_{3}(x, \varepsilon) & \geqslant \mathbb{P}^{x}\left(H(r) \leqslant u, X(t)<\varepsilon, R(t)<\varepsilon^{\nu}\right) \\
& \geqslant \mathbb{P}^{x}\left(H(r) \leqslant u, \int_{H(r)}^{t} R^{2}(v) \mathrm{d} v<\varepsilon-r^{2} u, R(t)<\varepsilon^{v}\right) .
\end{aligned}
$$

By the strong Markov property, this leads to:

$$
\begin{aligned}
p_{3}(x, \varepsilon) & \geqslant \int_{0}^{u} \mathbb{P}^{x}(H(r) \in \mathrm{d} s) \mathbb{P}^{r}\left(X(t-s)<\varepsilon-r^{2} u, R(t-s)<\varepsilon^{v}\right) \\
& =\int_{0}^{u} \mathbb{P}^{x}(H(r) \in \mathrm{d} s) \mathbb{P}^{r / \sqrt{t-s}}\left(X(1)<\frac{\varepsilon-r^{2} u}{(t-s)^{2}}, R(1)<\frac{\varepsilon^{v}}{\sqrt{t-s}}\right) \\
& \geqslant \mathbb{P}^{x}(H(r) \leqslant u) \inf _{s \in[0, u]} \mathbb{P}^{r / \sqrt{t-s}}\left(X(1)<\frac{\varepsilon-r^{2} u}{(t-s)^{2}}, R(1)<\frac{\varepsilon^{v}}{\sqrt{t-s}}\right) \\
& \geqslant \mathbb{P}^{x}(H(r) \leqslant u) \inf _{y \in[r / \sqrt{t}, r / \sqrt{t-u}]} \mathbb{P}^{y}\left(X(1)<\frac{\varepsilon-r^{2} u}{t^{2}}, R(1)<\frac{\varepsilon^{v}}{\sqrt{t}}\right) .
\end{aligned}
$$

This holds for all $u \in[0, t]$. We now choose $u=\chi \varepsilon$, for some constant $\chi \in\left(0, r^{-2}\right)$ whose value will be determined later. In view of Fact 2.1, we have 


$$
\begin{aligned}
\inf _{x \in[0, r]} \mathbb{P}^{x}(H(r) \leqslant u) & =\mathbb{P}(H(r) \leqslant u) \\
& =\mathbb{P}\left(\sup _{0 \leqslant v \leqslant u} R(v)>r\right) \\
& =\exp \left(-(1+o(1)) \frac{r^{2}}{2 \chi \varepsilon}\right), \quad \varepsilon \rightarrow 0,
\end{aligned}
$$

the last equality following from (2.17). On the other hand, Lemma 2.5 readily yields that, for our choice of $u$,

$$
\inf _{y \in[r / \sqrt{t}, r / \sqrt{t-u}]} \mathbb{P}^{y}\left(X(1)<\frac{\varepsilon-r^{2} u}{t^{2}}, R(1)<\frac{\varepsilon^{v}}{\sqrt{t}}\right)=\exp \left(-(1+o(1)) \frac{\left(r^{2}+t d\right)^{2}}{8\left(1-r^{2} \chi\right) \varepsilon}\right) .
$$

Assembling (2.32)-(2.34) gives

$$
\liminf _{\varepsilon \rightarrow 0} \inf _{x \in[0, r]} \varepsilon \log p_{3}(x, \varepsilon) \geqslant-\frac{r^{2}}{2 \chi}-\frac{\left(r^{2}+t d\right)^{2}}{8\left(1-r^{2} \chi\right)},
$$

for any $\chi \in\left(0, r^{-2}\right)$. Picking up $\chi=2 /\left(3 r^{2}+t d\right)$ completes the proof of Lemma 2.10.

Now we are ready to establish the main probability estimate which will be of use in the proof of Theorem 1.2.

Lemma 2.11. Fix $a \in(0,1), r>0, \delta \in\left(0, r^{2} / 3\right)$ and $v>\frac{1}{2}$. Let

$$
E_{6}(\varepsilon)=\left\{\sup _{0 \leqslant v \leqslant 1} R(v)<r, X(1)<\varepsilon, R(1)<\varepsilon^{v}, \sup _{s \in[a, 1]} R^{2}(s)>\sup _{s \in[0, a]} R^{2}(s)+\delta\right\} .
$$

Then

$$
\liminf _{\varepsilon \rightarrow 0} \varepsilon \log \mathbb{P}\left(E_{6}(\varepsilon)\right) \geqslant-\frac{(3 \delta+d)^{2}}{8}
$$

Proof. Let $b \in\left(0, r^{2} / 3-\delta\right)$ and $c \in(0,1)$. Note that

$$
\begin{gathered}
\mathbb{P}\left(E_{6}(\varepsilon)\right) \geqslant \mathbb{P}\left(\sup _{s \in[0, a]} R^{2}(s)<b, X(a)<c \varepsilon, b+\delta<\sup _{s \in[a, 1]} R^{2}(s)<r^{2},\right. \\
\left.X(1)-X(a)<(1-c) \varepsilon, R(1)<\varepsilon^{\nu}\right) .
\end{gathered}
$$

By the Markov property, this leads to:

$$
\mathbb{P}\left(E_{6}(\varepsilon)\right) \geqslant p_{4}(\varepsilon) \inf _{x \in[0, \sqrt{b}]} p_{5}(x, \varepsilon),
$$

where 
Favourite sites, favourite values and jump sizes

$$
\begin{aligned}
p_{4}(\varepsilon) & =\mathbb{P}\left(\sup _{s \in[0, a]} R^{2}(s)<b, X(a)<c \varepsilon\right), \\
p_{5}(x, \varepsilon) & =\mathbb{P}^{x}\left(b+\delta<\sup _{s \in[0,1-a]} R^{2}(s)<r^{2}, X(1-a)<(1-c) \varepsilon, R(1-a)<\varepsilon^{v}\right) .
\end{aligned}
$$

By Lemmas 2.3 and 2.6, for any $b_{1} \in(0, b)$,

$$
p_{4}(\varepsilon) \geqslant \mathbb{P}\left(b_{1} \leqslant \sup _{s \in[0, a]} R^{2}(s) \leqslant b, X(a)<c \varepsilon\right) \geqslant \exp \left(-(1+o(1)) \frac{\left(b_{1}+a d\right)^{2}}{8 c \varepsilon}\right),
$$

as $\varepsilon$ goes to 0 . Therefore

$$
p_{4}(\varepsilon) \geqslant \exp \left(-(1+o(1)) \frac{(b+a d)^{2}}{8 c \varepsilon}\right), \quad \varepsilon \rightarrow 0 .
$$

On the other hand, observe that

$$
\begin{aligned}
\inf _{x \in[0, \sqrt{b}]} p_{5}(x, \varepsilon) \geqslant & \inf _{x \in[0, \sqrt{b+\delta}]} \mathbb{P}^{x}\left(\sup _{s \in[0,1-a]} R^{2}(s)>b+\delta, X(1-a)<(1-c) \varepsilon, R(1-a)<\varepsilon^{v}\right) \\
& -\sup _{x \in(0, r]} \mathbb{P}^{x}\left(\sup _{s \in[0,1-a]} R^{2}(s) \geqslant r^{2}, X(1-a)<(1-c) \varepsilon\right) .
\end{aligned}
$$

Applying Lemmas 2.10 and 2.6 respectively to the two probability terms on the right-hand side yields

$$
\begin{aligned}
\inf _{x \in[0, \sqrt{b}]} p_{5}(x, \varepsilon) \geqslant & \exp \left(-(1+o(1)) \frac{(3(b+\delta)+(1-a) d)^{2}}{8(1-c) \varepsilon}\right) \\
& -\exp \left(-(1+o(1)) \frac{\left(r^{2}+(1-a) d\right)^{2}}{8(1-c) \varepsilon}\right)
\end{aligned}
$$

Since $3(b+\delta)<r^{2}$, this leads to

$$
\inf _{x \in[0, \sqrt{b}]} p_{5}(x, \varepsilon) \geqslant \exp \left(-(1+o(1)) \frac{(3(b+\delta)+(1-a) d)^{2}}{8(1-c) \varepsilon}\right) .
$$

Combining (2.35)-(2.37) yields that

$$
\liminf _{\varepsilon \rightarrow 0} \varepsilon \log \mathbb{P}\left(E_{6}(\varepsilon)\right) \geqslant-\frac{(b+a d)^{2}}{8 c}-\frac{(3(b+\delta)+(1-a) d)^{2}}{8(1-c)},
$$

for any $c \in(0,1)$ and $b \in\left(0, r^{2} / 3-\delta\right)$. Take $c=(b+a d) /(4 b+3 \delta+d)$ and then send $b$ to $0^{+}$to complete the proof of Lemma 2.11 . 


\section{Brownian local time}

Let $\{W(t) ; t \geqslant 0\}$ be a one-dimensional Brownian motion starting from 0 . Let $\{L(t, x)$; $t \geqslant 0, x \in \mathbb{R}\}$ denote its jointly continuous local time process, in the sense that, for any Borel function $f \geqslant 0$ and $t \geqslant 0$,

$$
\int_{0}^{t} f(W(s)) \mathrm{d} s=\int_{-\infty}^{\infty} f(x) L(t, x) \mathrm{d} x .
$$

For brevity, we write

$$
L^{*}(t)=\sup _{x \in \mathbb{R}} L(t, x), \quad t \geqslant 0
$$

For each $r>0$, define

$$
T_{r}=\inf \{t>0: W(t)>r\},
$$

the hitting time associated with $W$. Here is the key probability estimate in the proof of Theorem 1.1.

Lemma 3.1. Let $a \in(0,1), b_{2}>b_{1}>0, \rho>1$ and $0<c<(\rho-1) / b_{2}$. Let $\delta \in\left(0, b_{1}\right)$ be such that

$$
\sqrt{b_{2}}-\sqrt{b_{1}}>\sqrt{b_{1}}-\sqrt{b_{1}-\delta}
$$

We have

$$
\liminf _{\varepsilon \rightarrow 0} \varepsilon \log \mathbb{P}\left(F_{1}(\varepsilon)\right) \geqslant-\frac{\left(b_{1}-\delta+1\right)^{2}}{2}-\frac{5\left(\sqrt{b_{1}}-\sqrt{b_{1}-\delta}\right)^{2}}{c}
$$

where

$$
F_{1}(\varepsilon)=\left\{\varepsilon \leqslant T_{1} \leqslant \rho \varepsilon, b_{1} \leqslant L^{*}\left(T_{1}\right) \leqslant b_{2}, \sup _{x \in[a-c \varepsilon, a]} L\left(T_{1}, x\right)>\delta+\sup _{x \notin[a-c \varepsilon, a]} L\left(T_{1}, x\right)\right\} .
$$

Proof. The Ray-Knight theorem (Ray 1963; Knight 1963) says that $\left\{L\left(T_{1}, 1-x\right) ; x \geqslant 0\right\}$ is a continuous inhomogeneous Markov process. More precisely, when $x \in[0,1]$ it is a twodimensional squared Bessel process, starting from 0 , and when $x \geqslant 1$ it becomes a squared Bessel process of 'dimension 0'.

Let $\{R(t) ; t \geqslant 0\}$ be a two-dimensional Bessel process with $R(0)=0$, and $\{Z(t) ; t \geqslant 0\}$ a Bessel process of dimension 0 , with $Z(0)=1$. We assume that $R$ and $Z$ are independent. As before, we write

$$
X(t)=\int_{0}^{t} R^{2}(s) \mathrm{d} s, \quad t \geqslant 0
$$

Observe that $T_{1}=\int_{0}^{\infty} L\left(T_{1}, 1-x\right) \mathrm{d} x$. By the Ray-Knight theorem and the scaling property for the Bessel process (writing $x \vee y$ for $\max (x, y)$ ), 


$$
\begin{gathered}
\mathbb{P}\left(F_{1}(\varepsilon)\right)=\mathbb{P}\left(\varepsilon \leqslant X(1)+R^{4}(1) \int_{0}^{\infty} Z^{2}(s) \mathrm{d} s \leqslant \rho \varepsilon, b_{1} \leqslant \sup _{0 \leqslant s \leqslant 1} R^{2}(s) \vee R^{2}(1) \sup _{s \geqslant 0} Z^{2}(s) \leqslant b_{2},\right. \\
\left.\sup _{s \in J(\varepsilon)} R^{2}(s)>\delta+\sup _{s \in[0,1] \backslash J(\varepsilon)} R^{2}(s) \vee R^{2}(1) \sup _{s \geqslant 0} Z^{2}(s)\right),
\end{gathered}
$$

where $J(\varepsilon)=[1-a, 1-a+c \varepsilon]$. Let $\rho_{1} \in(1, \rho)$ be such that $c<\left(\rho_{1}-1\right) / b_{2}$. For all sufficiently small $\varepsilon>0$,

$$
\begin{aligned}
\mathbb{P}\left(F_{1}(\varepsilon)\right) \geqslant & \mathbb{P}\left(\int_{0}^{\infty} Z^{2}(s) \mathrm{d} s \leqslant 1, \sup _{s \geqslant 0} Z^{2}(s) \leqslant 2\right) \\
& \times \mathbb{P}\left(R(1)<\varepsilon^{v}, \varepsilon \leqslant X(1) \leqslant \rho_{1} \varepsilon, b_{1} \leqslant \sup _{0 \leqslant s \leqslant 1} R^{2}(s) \leqslant b_{2},\right. \\
& \left.\sup _{s \in J(\varepsilon)} R^{2}(s)>\delta+\sup _{s \in[0,1] \backslash J(\varepsilon)} R^{2}(s)\right) .
\end{aligned}
$$

The first probability term on the right-hand side is a positive constant. Lemma 3.1 now follows from Lemma 2.9.

In the proof of Theorem 1.2, we need two other probability estimates for Brownian local time. Their proofs are in the same spirit as the proof of Lemma 3.1, based on the RayKnight theorem and using Lemmas 2.11 and 2.10 respectively in lieu of Lemma 2.9. The details are omitted.

Lemma 3.2. Let $u \in(0,1), b>0$ and $\delta \in(0, b / 3)$. Then

$$
\liminf _{\varepsilon \rightarrow 0} \varepsilon \log \mathbb{P}\left(L^{*}\left(T_{1}\right)<b, T_{1}<\varepsilon, \sup _{x \in[0, u]} L\left(T_{1}, x\right)>\sup _{x>u} L\left(T_{1}, x\right)+\delta\right) \geqslant-\frac{(3 \delta+2)^{2}}{8} .
$$

Lemma 3.3. For $b>0$,

$$
\liminf _{\varepsilon \rightarrow 0} \varepsilon \log \mathbb{P}\left(L^{*}\left(T_{1}\right)>b, T_{1}<\varepsilon, \inf _{t \in\left[0, T_{1}\right]} W(t)>-1\right) \geqslant-\frac{(3 b+2)^{2}}{8} .
$$

\section{Theorem 1.2 and part (a) of Theorem 1.1}

In this section, we prove Theorem 1.2 and part (a) of Theorem 1.1. Let us first recall the following strong approximation theorem which holds simultaneously for local time and random walks. 
Fact 4.1. (Révész 1990, pp. 105-107). After possible redefinitions of variables and processes, there exists a coupling for the simple random walk $\left\{S_{k}\right\}_{k \geqslant 0}$ and the Wiener process $\{W(t) ; t \geqslant 0\}$, such that, for all $\varepsilon>0$, as $n$ goes to infinity,

$$
\begin{array}{r}
\sup _{x \in \mathbb{Z}} \max _{0 \leqslant k \leqslant n}|\xi(k, x)-L(k, x)|=o\left(n^{1 / 4+\varepsilon}\right) \text { a.s., } \\
\max _{0 \leqslant k \leqslant n}\left|S_{k}-W(k)\right|=\mathscr{O}(\log n) \text { a.s., }
\end{array}
$$

where $\xi(n, x)$ and $L(n, x)$ are defined in (1.1) and (3.1), respectively.

In the proof of Theorem 1.2 and of part (a) of Theorem 1.1, we shall be working in the probability space such that the coupling for $\left\{S_{n}\right\}_{n \geqslant 0}$ and $W$ in Fact 4.1 holds.

Proof of Theorem 1.2. Fix $\kappa \in(0,1)$, and define $r_{n}=r_{n}(\kappa)=\exp \left(n^{1+\kappa}\right)$. Let $T$ be the first hitting time process for $W$ (see (3.2)).

Let $u \in(0,0.01)$ be such that

$$
\frac{\left(u^{2}+2(1-u)\right)^{2}}{4}+49 u<\frac{1+2 \kappa}{1+\kappa} .
$$

Define

$$
W^{(n)}(t)=W\left(t+T_{r_{n}}\right)-r_{n}, \quad t \in\left[0, T_{r_{n+1}}-T_{r_{n}}\right] .
$$

By the strong Markov property, these are independent Brownian pieces. We can define $T_{r}^{(n)}=\inf \left\{t>0: W^{(n)}(t)>r\right\}, 0<\mathrm{r} \leqslant r_{n+1}-r_{n}$, the first hitting time process for $W^{(n)}$, and also $\left\{L^{(n)}(t, x) ; 0 \leqslant t \leqslant T_{r_{n+1}-r_{n}}^{(n)}, x \in \mathbb{R}\right\}$, the local time of $W^{(n)}$.

Consider the following measurable events:

$$
\begin{aligned}
A_{1, n}= & \left\{\sup _{x \in \mathbb{R}} L^{(n)}\left(T_{(1-u) r_{n+1}}^{(n)}, x\right)<u r_{n+1}, T_{(1-u) r_{n+1}}^{(n)}<\frac{(1+2 \kappa) r_{n+1}^{2}}{2 \log _{2} r_{n+1}},\right. \\
A_{2, n}= & \left.\left\{\sup _{x \in\left[0, u r_{n+1}\right]} L^{(n)}\left(T_{(1-u) r_{n+1}}^{(n)}, x\right)>\sup _{x>u r_{n+1}} L^{(n)}\left(T_{r_{n+1}-r_{n}}^{(n)}, x\right)-L_{(1-u) r_{n+1}}^{(n)}, x\right)+\frac{u^{2} r_{n+1}}{3}\right\}, \\
& \left.T_{r_{n+1}-r_{n}}^{(n)}-T_{(1-u) r_{n+1}}^{(n)}<\frac{\left.(1+2 \kappa) r_{n+1}, x\right)}{2 \log _{2} r_{n+1}}, x 2 u r_{n+1}^{2}, \underset{T_{(1-u) r_{n+1}}^{(n)} \leqslant t \leqslant T_{r_{n+1}-r_{n}}^{(n)}}{\operatorname{ing}} W^{(n)}(t)>(1-2 u) r_{n+1}\right\} .
\end{aligned}
$$

By the strong Markov property, $\left\{A_{1, n}, A_{2, n}\right\}_{n \geqslant 3}$ are mutually independent events. In particular, 
Favourite sites, favourite values and jump sizes

$$
\mathbb{P}\left(A_{1, n} \cap A_{2, n}\right)=\mathbb{P}\left(A_{1, n}\right) \mathbb{P}\left(A_{2, n}\right) .
$$

Since $\left\{W^{(n)}(t), t \in\left[0, T_{r_{n+1}}-T_{r_{n}}\right]\right\}$ is again a Brownian motion,

$$
\begin{gathered}
\mathbb{P}\left(A_{1, n}\right)=\mathbb{P}\left(\sup _{x \in \mathbb{R}} L\left(T_{(1-u) r_{n+1}}, x\right)<u r_{n+1}, T_{(1-u) r_{n+1}}<\frac{(1+2 \kappa) r_{n+1}^{2}}{2 \log _{2} r_{n+1}},\right. \\
\sup _{x \in\left[0, u r_{n+1}\right]} L\left(T_{(1-u) r_{n+1}}, x\right)>\sup _{x>u r_{n+1}} L\left(T_{\left.\left.(1-u) r_{n+1}, x\right)+\frac{u^{2} r_{n+1}}{3}\right)}\right) \\
=\mathbb{P}\left(\sup _{x \in \mathbb{R}} L\left(T_{1}, x\right)<\frac{u}{1-u}, T_{1}<\frac{1+2 \kappa}{2(1-u)^{2} \log _{2} r_{n+1}},\right. \\
\left.\sup _{x \in[0, u /(1-u)]} L\left(T_{1}, x\right)>\sup _{x>u /(1-u)} L\left(T_{1}, x\right)+\frac{u^{2}}{3(1-u)}\right),
\end{gathered}
$$

the second equality following from the scaling property. Applying Lemma 3.2 gives that, when $n$ goes to infinity,

$$
\mathbb{P}\left(A_{1, n}\right) \geqslant \exp \left(-(1+o(1)) \frac{\left(u^{2}+2(1-u)\right)^{2}}{4(1+2 \kappa)} \log _{2} r_{n+1}\right) .
$$

Similarly,

$$
\begin{aligned}
& \mathbb{P}\left(A_{2, n}\right) \\
& =\mathbb{P}\left(\sup _{x \in \mathbb{R}} L\left(T_{u r_{n+1}-r_{n}}, x\right) \geqslant 2 u r_{n+1}, T_{u r_{n+1}-r_{n}}<\frac{(1+2 \kappa) u r_{n+1}^{2}}{2 \log _{2} r_{n+1}}, \inf _{0 \leqslant t \leqslant T_{u r_{n+1}-r_{n}}} W(t)>-u r_{n+1} .\right) \\
& =\mathbb{P}\left(\sup _{x \in \mathbb{R}} L\left(T_{1}, x\right) \geqslant \frac{2 u r_{n+1}}{u r_{n+1}-r_{n}}, T_{1}<\frac{(1+2 \kappa) u r_{n+1}^{2}}{2\left(u r_{n+1}-r_{n}\right)^{2} \log _{2} r_{n+1}},\right. \\
& \left.\inf _{0 \leqslant t \leqslant T_{1}} W(t)>-\frac{u r_{n+1}}{u r_{n+1}-r_{n}}\right) \\
& \geqslant \mathbb{P}\left(\sup _{x \in \mathbb{R}} L\left(T_{1}, x\right) \geqslant 4, T_{1}<\frac{1+2 \kappa}{2 u \log _{2} r_{n+1}}, \inf _{0 \leqslant t \leqslant T_{1}} W(t)>-1\right),
\end{aligned}
$$

the last inequality following from the fact that $u r_{n+1}-r_{n} \in\left[u r_{n+1} / 2, u r_{n+1}\right]$ for all sufficiently large $n$. In the light of Lemma 3.3, this implies

$$
\mathbb{P}\left(A_{2, n}\right) \geqslant \exp \left(-(1+o(1)) \frac{49 u}{1+2 \kappa} \log _{2} r_{n+1}\right) .
$$

In view of (4.3)-(4.6), we have $\sum_{n} \mathbb{P}\left(A_{1, n} \cap A_{2, n}\right)=\infty$. By the Borel-Cantelli lemma, $\mathbb{P}\left(A_{1, n} \cap A_{2, n}\right.$, infinitely often (i.o.) $)=1$.

Now let us see what happens on $A_{1, n} \cap A_{2, n}$. First, we have, on $A_{1, n} \cap A_{2, n}$, 


$$
T_{r_{n+1}-r_{n}}^{(n)}<\frac{(1+2 \kappa)(1+u) r_{n+1}^{2}}{2 \log _{2} r_{n+1}}
$$

By definition, for $r \in\left(0, r_{n+1}-r_{n}\right]$ and $x \in \mathbb{R}$,

$$
\begin{aligned}
T_{r}^{(n)} & =T_{r+r_{n}}-T_{r_{n}}, \\
L^{(n)}\left(T_{r}^{(n)}, x\right) & =L\left(T_{r+r_{n}}, x+r_{n}\right)-L\left(T_{r_{n}}, x+r_{n}\right) .
\end{aligned}
$$

Writing

$$
\begin{aligned}
& s_{n}=(1-u) r_{n+1}+r_{n} \in\left(r_{n}, r_{n+1}\right), \\
& p_{n}=u r_{n+1}+r_{n} \in\left(r_{n}, s_{n}\right),
\end{aligned}
$$

we obtain, on $A_{1, n} \cap A_{2, n}$,

$$
\begin{aligned}
\sup _{y \in \mathbb{R}}\left(L\left(T_{S_{n}}, y\right)-L\left(T_{r_{n}}, y\right)\right)<u r_{n+1}, \\
\sup _{y \in\left[r_{n}, p_{n}\right]}\left(L\left(T_{S_{n}}, y\right)-L\left(T_{r_{n}}, y\right)\right)>\sup _{y>p_{n}}\left(L\left(T_{s_{n}}, y\right)-L\left(T_{r_{n}}, y\right)\right)+\frac{u^{2} r_{n+1}}{3}, \\
\sup _{y \in \mathbb{R}}\left(L\left(T_{r_{n+1}}, y\right)-L\left(T_{s_{n}}, y\right)\right) \geqslant 2 u r_{n+1}, \\
\inf _{T_{s_{n}} \leqslant t \leqslant T_{r_{n+1}}} W(t)>(1-2 u) r_{n+1}+r_{n} .
\end{aligned}
$$

On the other hand, by (4.7) and (4.8), we have, on $A_{1, n} \cap A_{2, n}$,

$$
T_{r_{n+1}}-T_{r_{n}}<\frac{(1+2 \kappa)(1+u) r_{n+1}^{2}}{2 \log _{2} r_{n+1}} \text {. }
$$

Since $\mathbb{P}\left(A_{1, n} \cap A_{2, n}\right.$ i.o. $)=1$, we know that there are almost surely infinitely many $n$ satisfying (4.10)-(4.14). We shall now only be considering these infinitely many $n$.

Since $r \mapsto T_{r}$ is a subordinator of index $\frac{1}{2}$, we have (Fristedt 1974): for any $\varepsilon \in(0,0.01)$, almost surely for large $r$,

$$
T_{r}<r^{2+\varepsilon}
$$

Let $\xi$ be the local time of the random walk $\left\{S_{k}\right\}_{k \geqslant 0}$ satisfying (4.1) and (4.2). In view of (4.1), almost surely for large $n,(4.10)$ implies (recalling $\xi^{*}(n)$ from (1.5))

$$
\xi^{*}\left(\left\lfloor T_{s_{n}}\right\rfloor\right) \leqslant u r_{n+1}+T_{s_{n}}^{1 / 4+\varepsilon}+\xi^{*}\left(\left\lfloor T_{r_{n}}\right\rfloor\right)+T_{r_{n}}^{1 / 4+\varepsilon},
$$

which, in light of (4.15) and (1.6), yields

$$
\xi^{*}\left(\left\lfloor T_{S_{n}}\right\rfloor\right) \leqslant(1+\varepsilon) u r_{n+1},
$$

whereas (4.12) implies

$$
\xi^{*}\left(\left\lfloor T_{r_{n+1}}\right\rfloor\right) \geqslant(2-\varepsilon) u r_{n+1} .
$$


By the definition of favourite sites, these two estimates imply that, $U\left(\left\lfloor T_{r_{n+1}}\right\rfloor\right)$, the favourite site at time $\left\lfloor T_{r_{n+1}}\right\rfloor$, must be visited by the random walk during $\left(\left\lfloor T_{S_{n}}\right\rfloor,\left\lfloor T_{r_{n+1}}\right\rfloor\right.$, i.e.,

$$
U\left(\left\lfloor T_{r_{n+1}}\right\rfloor\right)=S_{j}, \quad \text { for some } j=j_{n}(\omega) \in\left(\left\lfloor T_{S_{n}}\right\rfloor,\left\lfloor T_{\left.r_{n+1}\right\rfloor}\right\rfloor\right] .
$$

On the other hand, (4.13) and (4.2) together confirm that

$$
S_{i}>(1-2 u) r_{n+1}, \quad \text { for all } i \in\left[\left\lfloor T_{S_{n}}\right\rfloor,\left\lfloor T_{r_{n+1}}\right\rfloor\right] .
$$

Now consider (4.11). Again, by means of (4.1), (4.15) and (1.6), it follows from (4.11) that

$$
\max _{y \in\left[r_{n}, p_{n}\right]} \xi\left(\left\lfloor T_{S_{n}}\right\rfloor, y\right)>\max _{y>p_{n}} \xi\left(\left\lfloor T_{S_{n}}\right\rfloor, y\right)+\left(\frac{1}{3}-\varepsilon\right) u^{2} r_{n+1},
$$

which, by definition, implies $U\left(\left\lfloor T_{S_{n}}\right\rfloor\right) \leqslant p_{n}$. Since $p_{n}<(1-2 u) r_{n+1}$, comparing this with (4.16) and (4.17), we conclude that there exists a random integer $m=m_{n}(\omega) \in\left[\left\lfloor T_{S_{n}}\right\rfloor\right.$, $\left.\left\lfloor T_{r_{n+1}}\right\rfloor\right]$ such that $U(m)=U\left(\left\lfloor T_{S_{n}}\right\rfloor\right)$ and that $U(m+1)>(1-2 u) r_{n+1}$. For this $m$,

$$
U(m+1)-U(m)>(1-2 u) r_{n+1}-p_{n} \geqslant(1-4 u) r_{n+1},
$$

and, of course, $m \in\left[T_{r_{n}}-1, T_{r_{n+1}}\right]$.

To complete the proof of Theorem 1.2, it suffices to note that, by (4.14) and (4.15), $T_{r_{n+1}}<(1+3 \kappa)(1+u) r_{n+1}^{2} / 2 \log _{2} r_{n+1}$, which implies

$$
r_{n+1}>\sqrt{\frac{2 T_{r_{n+1}} \log _{2} T_{r_{n+1}}}{(1+4 \kappa)(1+u)}} \geqslant \sqrt{\frac{2 m \log _{2} m}{(1+4 \kappa)(1+u)}} .
$$

Going back to (4.18), we see that

$$
U(m+1)-U(m)>(1-4 u) \sqrt{\frac{2 m \log _{2} m}{(1+4 \kappa)(1+u)}} .
$$

We have therefore proved that

$$
\limsup _{n \rightarrow \infty} \frac{U(n+1)-U(n)}{\sqrt{2 n \log _{2} n}} \geqslant \frac{1-4 u}{\sqrt{(1+4 \kappa)(1+u)}} \text { a.s. }
$$

Since both $u$ and $\kappa$ can be as close to 0 as possible, this yields the lower bound in Theorem 1.2 .

The upper bound is trivial. Indeed, $U(n+1)-U(n) \leqslant \max _{0 \leqslant k \leqslant n} S_{k}-\min _{0 \leqslant k \leqslant n} S_{k}$, and the upper bound immediately follows from the usual LIL for the range of random walk (see Révész 1990, p. 44).

Proof of part (a) of Theorem 1.1. Again, let us fix $\kappa \in(0,1)$, and define $r_{n}=\exp \left(n^{1+\kappa}\right)$. Recall $T$ from (3.2). Let $a \in(0,1)$ and $b>0$.

Let $\rho \geq 1, b^{*}>b$ and $0<c<(\rho-1) / b^{*}$. We can choose $\delta \in(0, b)$ such that $\sqrt{b^{*}}-\sqrt{b}>\sqrt{b}-\sqrt{b-\delta}$ and such that 


$$
\frac{(b-\delta+1)^{2}}{2}+\frac{5(\sqrt{b}-\sqrt{b-\delta})^{2}}{c}<(1+\kappa) \frac{(b+1)^{2}}{2} .
$$

Fix $\gamma>0$ and let

$$
\begin{aligned}
I_{n} & =\left[a r_{n}-\frac{c \gamma r_{n}}{\log _{2} r_{n}}, a r_{n}\right], \\
A_{n} & =\left\{\frac{\gamma r_{n}^{2}}{\log _{2} r_{n}} \leqslant T_{r_{n}} \leqslant \frac{\rho \gamma r_{n}^{2}}{\log _{2} r_{n}}, b r_{n} \leqslant L^{*}\left(T_{r_{n}}\right) \leqslant b^{*} r_{n}, \sup _{x \in I_{n}} L\left(T_{r_{n}}, x\right)>\delta r_{n}+\sup _{x \notin I_{n}} L\left(T_{r_{n}}, x\right)\right\} .
\end{aligned}
$$

By scaling, Lemma 3.1 and (4.19), for all sufficiently large $n$,

$$
\mathbb{P}\left(A_{n}\right) \geqslant \exp \left(-(1+\kappa) \frac{(b+1)^{2} \log _{2} r_{n}}{2 \gamma}\right) .
$$

Therefore, as long as $\gamma \geqslant(1+\kappa)^{2}(b+1)^{2} / 2$, we have $\sum_{n} \mathbb{P}\left(A_{n}\right)=\infty$. Rigorously speaking, we cannot directly apply the Borel-Cantelli lemma here, since the events $\left(A_{n}\right)$ are not independent. However, as in the proof of Theorem 1.2, we can consider the independent Brownian pieces $W^{(n)}$ and construct events which are based on $W^{(n)}$, and use the same argument to get rid of the dependence difficulty. We choose to omit tedious discussions which are much like those in the proof of Theorem 1.2, and claim that $\mathbb{P}\left(A_{n}\right.$ i.o. $)=1$. Therefore, almost surely there exist infinitely many $n$ such that simultaneously

$$
\frac{\gamma r_{n}^{2}}{\log _{2} r_{n}} \leqslant T_{r_{n}} \leqslant \frac{\rho \gamma r_{n}^{2}}{\log _{2} r_{n}}, \quad b r_{n} \leqslant L^{*}\left(T_{r_{n}}\right) \leqslant b^{*} r_{n}, \quad \sup _{x \in I_{n}} L\left(T_{r_{n}}, x\right)>\delta r_{n}+\sup _{x \notin I_{n}} L\left(T_{r_{n}}, x\right) .
$$

Applying Fact 4.1 implies that, infinitely often,

$$
\frac{\gamma r_{n}^{2}}{\log _{2} r_{n}} \leqslant T_{r_{n}} \leqslant \frac{\rho \gamma r_{n}^{2}}{\log _{2} r_{n}}, \quad(1-\kappa) b r_{n} \leqslant \xi^{*}\left(\left\lfloor T_{r_{n}}\right\rfloor\right) \leqslant(1+\kappa) b^{*} r_{n}, \quad U\left(\left\lfloor T_{r_{n}}\right\rfloor\right) \in I_{n} .
$$

When $\gamma r_{n}^{2} / \log _{2} r_{n} \leqslant T_{r_{n}} \leqslant \rho \gamma r_{n}^{2} / \log _{2} r_{n}$, we have (recalling $\phi$ from (1.4))

$$
\begin{aligned}
b r_{n} & \geqslant \frac{(1+o(1)) b}{\sqrt{2 \rho \gamma}} \phi\left(T_{r_{n}}\right), \\
b^{*} r_{n} & \leqslant \frac{(1+o(1)) b^{*}}{\sqrt{2 \gamma}} \phi\left(T_{r_{n}}\right), \\
I_{n} & \subset\left[\frac{(1+o(1)) a}{\sqrt{2 \rho \gamma}} \phi\left(T_{r_{n}}\right), \frac{(1+o(1)) a}{\sqrt{2 \gamma}} \phi\left(T_{r_{n}}\right)\right] .
\end{aligned}
$$

Thus, for any $\kappa \in(0,1), b^{*}>b>0, \rho>1$ and $\gamma \geqslant(1+\kappa)^{2}(b+1)^{2} / 2$, with probability one, the random sequence $\left\{\left(U(n) / \phi(n), \xi^{*}(n) / \phi(n)\right)\right\}_{n \geqslant 3}$ visits

$$
\left[\frac{(1-\kappa) a}{\sqrt{2 \rho \gamma}}, \frac{(1+\kappa) a}{\sqrt{2 \gamma}}\right] \times\left[\frac{(1-\kappa)^{2} b}{\sqrt{2 \rho \gamma}}, \frac{(1+\kappa)^{2} b^{*}}{\sqrt{2 \gamma}}\right]
$$


infinitely often. Take $\gamma=(1+\kappa)^{2}(b+1)^{2} / 2$. Since $\left(\kappa, b^{*}, \rho\right)$ can be as close to $(0, b, 1)$ as possible, we conclude that $(a /(b+1), b /(b+1))$ is almost surely a limit point of $\left\{\left(U(n) / \phi(n), \xi^{*}(n) / \phi(n)\right)\right\}_{n \geqslant 3}$. For any $x>0$ and $y>0$ with $x+y<1$, we can choose $a \in(0,1)$ and $b>0$ such that $(x, y)=(a /(b+1), b /(b+1))$. Thus $(x, y)$ is a limit point of $\left\{\left(U(n) / \phi(n), \xi^{*}(n) / \phi(n)\right)\right\}_{n \geqslant 3}$. Of course, the positivity of $x$ is irrelevant, since when $x<0$, we can consider $\left\{-S_{n}\right\}_{n \geqslant 0}$ instead of $\left\{S_{n}\right\}_{n \geqslant 0}$.

As a consequence, any $(x, y) \in \mathscr{C}$ is almost surely a limit point of $\{(U(n) / \phi(n)$, $\left.\left.\xi^{*}(n) / \phi(n)\right)\right\}_{n \geqslant 3}$. We can interchange the order of 'any $(x, y) \in \mathscr{C}$ ' and 'almost surely' by considering the points in $\mathscr{b}$ whose coordinates are rationals.

\section{Proof of part (b) of Theorem 1.1}

Part (b) of Theorem 1.1 is a consequence of the following:

Theorem 5.1. For any $a \in(0,1)$,

$$
\limsup _{n \rightarrow \infty} \sup _{x \geqslant a \phi(n)} \frac{\xi(n, x)}{\phi(n)}=1-a \text { a.s. }
$$

More precisely, part (b) of Theorem 1.1 follows from the upper bound in Theorem 5.1.

In view of (4.1), we can equivalently state Theorem 5.1 as follows.

Theorem 5.2. Let $\{L(t, x) ; t \geqslant 0, x \in \mathbb{R}\}$ be a Brownian local time process, and let $a \in(0,1)$. Then

$$
\limsup _{t \rightarrow \infty} \sup _{x \geqslant a \phi(t)} \frac{L(t, x)}{\phi(t)}=1-a \text { a.s. }
$$

The main ingredient in the proof of Theorem 5.2 is the following tail estimate:

Lemma 5.3. For $a>0$ and $b>0$,

$$
\lim _{t \rightarrow \infty} \frac{1}{\log _{2} t} \log \mathbb{P}\left(\sup _{x \geqslant a \phi(t)} L(t, x)>b \phi(t)\right)=-(a+b)^{2} .
$$

The proof of Theorem 5.2 relies on Lemma 5.3 and the Borel-Cantelli lemma, along the same lines as in Section 4. No new ingredient is needed. So we feel free to omit the details. The rest of the section is devoted to the proof of Lemma 5.3.

Proof of Lemma 5.3. Let $T$ be the first hitting time process of the Brownian motion whose local time is $L$; see (3.2). By the strong Markov property, for any $u>0$ and $v>0$, 


$$
\begin{aligned}
\mathbb{P}\left(\sup _{x \geqslant u} L(t, x)>v\right) & =\int_{0}^{t} \mathbb{P}\left(T_{u} \in \mathrm{d} s\right) \mathbb{P}\left(\sup _{x \geqslant 0} L(t-s, x)>v\right) \\
& =\int_{0}^{t} \frac{u \mathrm{~d} s}{\sqrt{2 \pi s^{3}}} \exp \left(-\frac{u^{2}}{2 s}\right) \mathbb{P}\left(\sup _{x \geqslant 0} L(1, x)>\frac{v}{\sqrt{t-s}}\right) .
\end{aligned}
$$

It is known (see Kesten 1965; Csáki 1989) that

$$
\mathbb{P}\left(\sup _{x \geqslant 0} L(1, x)>\lambda\right)=\exp \left(-(1+o(1)) \frac{\lambda^{2}}{2}\right), \quad \lambda \rightarrow \infty .
$$

Therefore,

$$
\mathbb{P}\left(\sup _{x \geqslant a \phi(t)} L(t, x)>b \phi(t)\right)=\int_{0}^{t} \frac{a \phi(t) \mathrm{d} s}{\sqrt{2 \pi s^{3}}} \exp \left(-\frac{(a \phi(t))^{2}}{2 s}-(1+o(1)) \frac{(b \phi(t))^{2}}{2(t-s)}\right) .
$$

This completes the proof of Lemma 5.3 by means of an elementary argument in the spirit of Laplace's method (see, for example, Widder 1941).

\section{Acknowledgements}

The authors are indebted to the referees and an associate editor for helpful remarks. The first and second authors' research was supported in part by the Hungarian National Foundation for Scientific Research, grants nos T 019346 and T 029621. Cooperation between the authors was supported by the joint French-Hungarian Intergovernmental Grant 'Balaton' (grant no. F25/97).

\section{References}

Abramowitz, M. and Stegun, I.A. (1965) Handbook of Mathematical Functions. New York: Dover. Bass, R.F. and Griffin, P.S. (1985) The most visited site of Brownian motion and simple random walk. Z. Wahrscheinlichkeitstheorie Verw. Geb., 70, 417-436.

Bingham, N.H., Goldie, C.M. and Teugels, J.L. (1987) Regular Variation. Cambridge: Cambridge University Press.

Csáki, E. (1989) An integral test for the supremum of Wiener local time. Probab. Theory Related Fields, 83, 207-217.

Csáki, E. and Shi, Z. (1998) Large favourite sites of simple random walk and the Wiener process. Electron. J. Probab., 3(14).

Eisenbaum, N. (1989) Temps locaux, excursions et lieu le plus visité par un mouvement brownien linéaire. Doctoral thesis, Université Paris VII.

Eisenbaum, N. (1997) On the most visited sites by a symmetric stable process. Probab. Theory Related Fields, 107, 527-535.

Erdős, P. and Révész, P. (1984) On the favourite points of a random walk. In B. Sendov (ed.), Mathematical Structures - Computational Mathematics - Mathematical Modelling, Vol. 2, pp. 
152-157. Sofia: Bulgarian Academy of Sciences.

Erdős, P. and Révész, P. (1987) Problems and results on random walks. In P. Bauer, F. Konecny and W. Wertz (eds), Mathematical Statistics and Probability Theory, Vol. B, pp. 59-65. Dordrecht: Reidel.

Fristedt, B. (1974) Sample functions of stochastic processes with stationary independent increments. In P.E. Ney and S.C. Port (eds), Advances in Probability and Related Topics, Vol. 3, pp. 241-396. New York: Dekker.

Kesten, H. (1965) An iterated logarithm law for the local time. Duke Math. J., 32, 447-456.

Knight, F.B. (1963) Random walks and the sojourn density process of Brownian motion. Trans. Amer. Math. Soc., 109, 56-86.

Leuridan, C. (1997) Le point d'un fermé le plus visité par le mouvement brownien. Ann. Probab., 25, 953-996.

Marcus, M.B. and Shepp, L.A. (1972) Sample behavior of Gaussian processes. In L. LeCam, J. Neyman and E.L. Scott (eds), Proceedings of the Sixth Berkeley Symposium on Mathematical Statistics and Probability, Vol. 2, pp. 423-441. Berkeley: University of California Press.

Pitman, J.W. and Yor, M. (1982) A decomposition of Bessel bridges. Z. Wahrscheinlichkeitstheorie Verw. Geb., 59, 425-457.

Ray, D. (1963) Sojourn times of a diffusion process. Illinois J. Math., 7, 615-630.

Révész, P. (1990) Random Walk in Random and Non-random Environments. Singapore: World Scientific.

Revuz, D. and Yor, M. (1999) Continuous Martingales and Brownian Motion, 3rd edn. Berlin: Springer-Verlag.

Tóth, B. and Werner, W. (1997) Tied favourite edges for simple random walk. Combin. Probab. Comput., 6, 359-369.

Widder, D.V. (1941) The Laplace Transform. Princeton, NJ: Princeton University Press.

Received March 1999 and revised November 1999 